\title{
A construção do campo da Saúde Coletiva e as políticas de saúde - Contribuições da Revista Ciência \& Saúde Coletiva
}

\author{
The building of Collective Health and health policies \\ - Contributions from Journal Ciência \& Saúde Coletiva
}

Ligia Maria Vieira-da-Silva (http://orcid.org/0000-0003-2518-411X) ${ }^{1}$

Monique Azevedo Esperidião (https://orcid.org/0000-0003-1827-3595) ${ }^{1}$

Anne Soares Silveira (https://orcid.org/0000-0001-5982-052X) ${ }^{1}$

Jairnilson Silva Paim (http://orcid.org/0000-0003-0783-262X) ${ }^{1}$
${ }^{1}$ Instituto de Saúde Coletiva, Universidade Federal da Bahia. R. Basílio da Gama s/n, Canela. 40110-040 Salvador BA Brasil.ligiamvs@ufba.br

\begin{abstract}
This paper aimed to analyze the contribution of Journal Ciência \& Saúde Coletiva (C\&SC) to the construction of the CH field, particularly in its relationships with the Health Policy, understood both as an academic discipline and as a scope of practice. We reviewed papers published between 1996 and 2019 in the CeSC. Titles and abstracts of the 397 documents on health policies were read to measure the magnitude and identify the main themes and theoretical-methodological approaches. Thirty-five documents were selected and read in full among the 142 revised ones to investigate the Journal's contribution to CH's construction. The analysis was based on Bourdieu's sociology. It revealed that $\mathrm{C} \triangleleft \mathrm{SC}$ was established as a space for the construction of $\mathrm{CH}$ in multiple dimensions, particularly concerning the reflexivity on the field. Specifically, concerning the Health Policy, despite the small percentage of documents on the issue (6.8\%), it encompassed the different meanings of this subject for the field. The authors discuss the possible relationships between the characteristics identified and the historical process of incorporating this subject in the various areas of $\mathrm{CH}$.
\end{abstract}

Key words Health policy, Health policies, Public policies, Collective health
Resumo Com o objetivo de analisar a contribuição da Revista Ciência « Saúde Coletiva (CひSC) para a construção do campo da Saúde Coletiva, particularmente nas suas relações com a Política de Saúde, compreendida tanto como disciplina acadêmica como âmbito de práticas, foi realizada uma revisão dos artigos publicados na CહSC no periodo compreendido entre 1996 e 2019. Para dimensionar a magnitude e identificar as principais temáticas e abordagens teórico-metodológicas foram lidos títulos e resumos de 397 documentos sobre políticas de saúde. Já para investigar a contribuição da Revista para a construção do campo da Saúde Coletiva foram selecionados e lidos na integra 35 documentos dentre os 142 localizados. A análise apoiou-se na sociologia de Bourdieu. Revelou que a CerSC constituiu-se em um dos espaços de construção da Saúde Coletiva em múltiplos âmbitos, particularmente no que diz respeito à reflexividade sobre campo. Especificamente em relação à Política de Saúde, a despeito do pequeno percentual de documentos sobre essa problemática (6,8\%), ela abarcou os diversos significados dessa temática para o campo. Os autores discutem as possiveis relações existentes entre as características identificadas e o processo histórico de incorporação desse objeto nos diversos âmbitos de constituição do campo da Saúde Coletiva.

Palavras-chave Politica de saúde, Politicas de saúde, Políticas públicas, Saúde coletiva 


\section{Introdução}

As políticas de saúde têm sido estudadas tanto por pesquisadores que a consideram como uma disciplina estabelecida nos países industrializados ${ }^{1,2}$ como por organizações privadas, denominadas think-tanks, situadas entre as ciências sociais acadêmicas, o governo e os partidos políticos ${ }^{3}$.

No Brasil, essa temática foi desenvolvida principalmente ao interior da Saúde Coletiva (SC), originada nos anos 70 do século XX, e que também incorpora essa dupla dimensão, podendo ser compreendida como campo de saberes e âmbito de práticas ${ }^{4-6}$.

Embora a SC tenha sido investigada sob diversos enfoques, há um razoável consenso acerca da adequação do uso da abordagem de campo, no sentido de Bourdieu, para interpretá-la $\mathrm{a}^{5,7-14}$.

O recurso a esse referencial teórico mostrou que a SC na sua emergência, poderia ser considerada como um espaço social com um propósito de tornar-se campo. Já seu desenvolvimento subsequente permitiu considerá-la um campo em processo de consolidação ${ }^{15}$, o que se traduz na sua institucionalização em diversos âmbitos sociais $^{16}$, particularmente no campo científico, com o desenvolvimento da sua Pós-Graduação ${ }^{17}$ e da ampliação da sua produção acadêmica indexada em bases internacionais ${ }^{18,19}$. Por outro lado, as relações entre a SC e a Reforma Sanitária Brasileira ${ }^{20}$ revelam a influência da racionalidade do campo político.

Nessa interface, a análise política e das políticas aparece com destaque na constituição da SC, permeando todos os principais subcampos, a saber, a Epidemiologia, as Ciências Sociais e Humanas em Saúde, ainda que seja central para a área denominada de Políticas, Planejamento e Gestão ${ }^{21}$.

Também, ao longo dos últimos 20 anos verificou-se uma tendência à especialização ao interior da SC que se traduz pela organização dos grupos de trabalho vinculados à Associação Brasileira de Saúde Coletiva (Abrasco). Para cada temática, além de estudos epidemiológicos ou de abordagens orientadas pelas ciências humanas e sociais, há a discussão das políticas específicas.

Os periódicos que veiculam a produção da SC brasileira, têm sido analisados no que diz respeito à sua contribuição para a Saúde Pública internacional ${ }^{22}$, à institucionalização da $\mathrm{SC}^{16}$, à sua internacionalização ${ }^{19}$, bem como têm sido objeto de autoanálise por parte dos editores ${ }^{14,23-30}$.

A vinculação da Revista Ciência \& Saúde Coletiva (C\&SC) em relação à Abrasco justifica uma investigação específica buscando identificar qual a sua contribuição para a constituição da SC, particularmente no que diz respeito às políticas de saúde, aspecto central da interface entre conhecimento e práticas. Como objeto de conhecimento aparece tanto articulada às ciências sociais em saúde como à área denominada Política, Planejamento e Gestão em Saúde (PP\&GS).

Por outro lado, a caracterização da produção de artigos sobre políticas de saúde na C\&SC no que diz respeito a sua magnitude, tipos de artigos e abordagens teórico-metodológicas, tem sido secundarizada bem como a discussão acerca das suas relações com a construção da SC. Assim, o presente artigo tem por objetivo analisar possíveis contribuições da C\&SC na divulgação da produção científica e técnica em Política de Saúde e na constituição do campo da SC.

\section{Metodologia}

Foram inicialmente revisados artigos que tratavam especificamente da problemática do campo da SC, artigos e números temáticos voltados para a discussão da Pós-Graduação, além de temas relacionados com a natureza do conhecimento, bem como sobre a constituição dos principais subespaços da SC: Epidemiologia, Ciências Humanas e Sociais em Saúde e a área de Política, Planejamento e Gestão em Saúde. Com essa finalidade, foi realizada uma revisão dos artigos publicados no período compreendido entre 1996 e 2019. Foi feita a busca no site da Revista por meio das seguintes palavras-chave, apenas no título, de forma sucessiva: campo; saúde coletiva; pós-graduação e Bourdieu. Foram identificados 244 artigos. Após leitura dos títulos e eliminação de artigos que não tratavam especificamente da temática restaram 146. Foram lidos os resumos e 35 artigos na íntegra.

A análise das relações entre a produção de artigos em políticas e a construção do campo da SC na Revista foi orientada pela sociologia de Bourdieu. O conceito de campo para esse autor corresponde a um microcosmo social, uma rede de relações objetivas entre posições, agentes e instituições, dotado de autonomia relativa no qual existem lutas específicas que fazem sentido para os seus integrantes ${ }^{31}$. Já o conceito de espaço social é usado por Bourdieu ou como sinônimo de campo ou por referência ao espaço social global que, por sua vez, seria constituído por campos. Também como microcosmo social mais delimitado, intercampos, onde agentes oriundos 
de âmbitos distintos interagem movidos por um interesse em comum ${ }^{32}$. Para esse autor um campo é uma construção teórica que é simultaneamente um espaço de forças e um espaço de lutas ${ }^{31}$. Nesse sentido, as políticas de saúde podem ser analisadas como parte de um dos subespaços da Saúde Coletiva, a saber, aquele denominado de Políticas, Planejamento e Gestão em Saúde, que corresponde a um dos polos desse campo ${ }^{15}$. Como tema de investigação interdisciplinar, suas abordagens metodológicas e teóricas podem ser objeto de disputas entre os outros subespaços da SC e outros campos.

Para a caracterização dos artigos sobre políticas de saúde foram usadas as seguintes palavras-chave: políticas; política; políticas de saúde; Sistema Único de Saúde; SUS; Reforma Sanitária; reformas; privado; financiamento. Do total de 806 artigos assim selecionados foram descartadas 253 duplicatas tendo restado 553 artigos. Em seguida, foi feita a leitura dos títulos e resumos e descartados 156, restando 397 documentos (Figura 1).

Os artigos incluídos foram classificados segundo a seguinte tipologia de áreas e subáreas temáticas ${ }^{33}$ : a) análise política em saúde; b) componentes dos sistemas de saúde; c) análise de políticas específicas de saúde. Os estudos sobre avaliação de programas foram excluídos e somente revisados aqueles que tinham por objeto a avaliação de políticas.
Os estudos classificados como 'Análise política em saúde ${ }^{34}$ investigam as relações de poder em saúde (natureza, estrutura, relações, distribuição e lutas), bem como o processo político em saúde e suas relações com a produção de fatos políticos, incluindo os estudos de conjunturas. Tal perspectiva considera o poder como categoria central, analisando sua apropriação, distribuição e disputa nos âmbitos setorial e societário ${ }^{34}$. Os trabalhos agrupados como 'Análise de políticas específicas de saúde', por sua vez, tratam do conteúdo das políticas enquanto diretrizes, planos e programas $^{35}$, compreendendo o estudo de políticas específicas, como políticas voltadas a grupos populacionais (mulher, criança, idoso, trabalhador etc.) ou ao enfrentamento de problemas (Aids, dengue, hipertensão arterial, tuberculose etc.). A categoria "Componentes do Sistema de Saúde" inclui os estudos que abordam a dinâmica política em torno dos diversos elementos constitutivos do sistema, tais como financiamento; gestão; participação e controle social; modelos de atenção; recursos humanos; ciência, tecnologia e inovação $0^{33}$.

Foi feita também uma codificação da abordagem metodológica segundo a nomenclatura explicitada pelo autor no resumo em: estudos de caso; estudos de casos múltiplos; estudos sócio-históricos; análise de implantação; análise da formulação de política; análise exploratória; estudos qualitativos; artigos de revisão; ensaios;

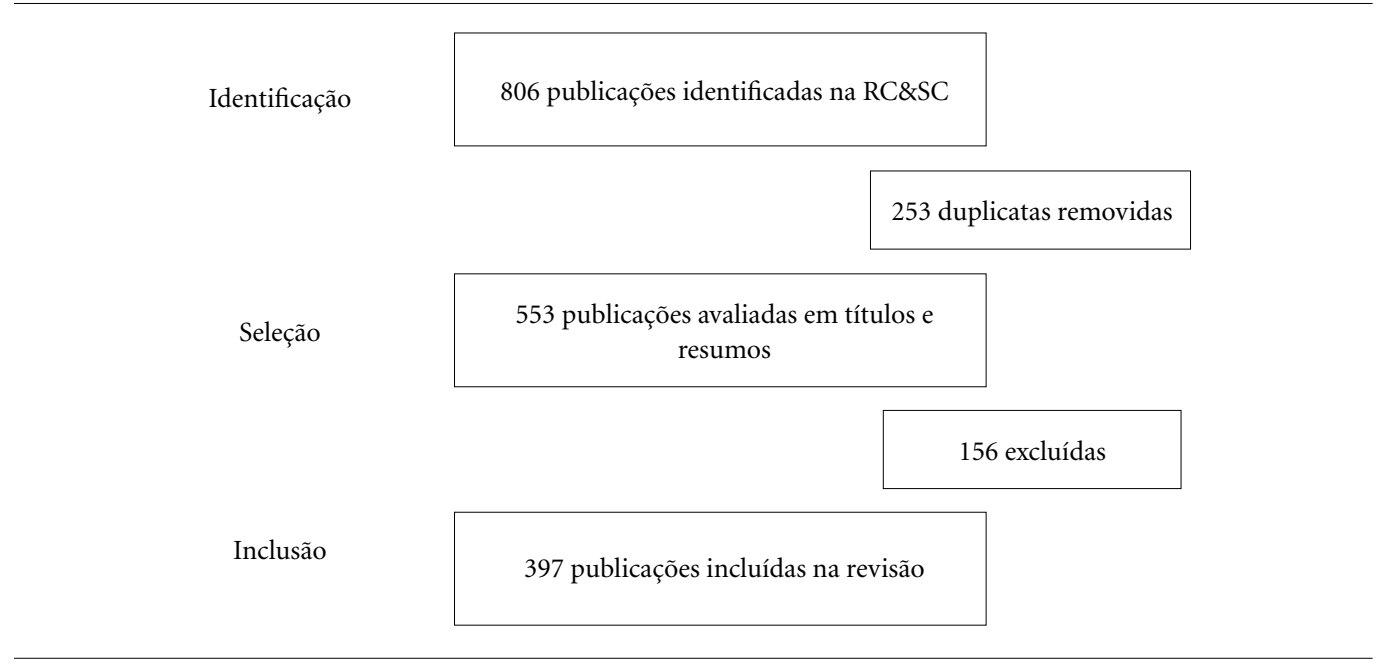

Figura 1. Fluxograma relativo ao processo de seleção dos documentos sobre políticas de saúde publicados pela Revista Ciência \& Saúde Coletiva (1996-2019) 
métodos e técnicas não explicitados; outros. Para os editoriais e resenhas utilizou-se a codificação "não se aplica". Os referenciais teóricos, quando explicitados nos resumos, foram mantidos conforme designação dos autores.

\section{Resultados e discussão}

\section{As políticas de saúde na C\&SC}

A produção sobre políticas de saúde encontrada na C\&SC no período 1996 a 2019 totalizou 397 trabalhos. Entre eles, a maioria é composta por artigos $(53,7 \%)$, seguidos de debates $(16,6 \%)$ e Temas Livres (13,6\%) (Tabela 1). Considerando um total de 5.871 documentos publicados pela Revista no período, a produção encontrada corresponde a 6,8\%. Comparando especificamente o percentual de artigos, a Revista publicou no período 5.033 artigos, sendo 213 de políticas de saúde $(4,2 \%)$. Esse pequeno percentual pode estar relacionado com as escolhas editoriais ou com características da produção da área.

A análise temática revelou que 40,3\% dos trabalhos abordam aspectos referentes aos Componentes do Sistema de Saúde; seguida de 31,5\% classificados como Análise de Políticas de Saúde Específicas e 28,2\% como Análise Política em Saúde (Tabela 2). Este achado diverge dos resultados encontrados por Santos e Teixeira ${ }^{33}$, que identificaram um predomínio de estudos na área Análise de Políticas de Saúde Específicas.

Entretanto, entre o elenco de subtemas especificados, o maior número de publicações encontrados na presente pesquisa refere-se às políticas voltadas para o enfrentamento de problemas es-

Tabela 1. Número e percentual de textos publicados na Revista Ciência \& Saúde Coletiva sobre políticas de saúde segundo tipo de publicação (1996-2019).

\begin{tabular}{lrr}
\hline Tipo de publicação & $\mathbf{N}$ & $\%$ \\
\hline Artigo & 213 & 53,7 \\
Carta & 1 & 0,3 \\
Debate & 66 & 16,6 \\
Editorial & 18 & 4,5 \\
Opinião & 20 & 5,0 \\
Resenha & 13 & 3,3 \\
Revisão & 12 & 3,0 \\
Temas livres & 54 & 13,6 \\
Total & 397 & 100,0 \\
\hline
\end{tabular}

Fonte: Revista Ciencia \& Saúde Coletiva. Homepage Scielo. Disponível em: https://www.scielo.br/scielo.php?script=sci_ serial\&pid=1413-8123\&lng=en pecíficos $(23,9 \%)$, seguidos de gestão de sistemas $(13,1 \%)$ e processo de construção do SUS (10\%). Nos estudos sobre Análise Política em Saúde observa-se um aumento do número de trabalhos sobre as relações entre o público e o privado (6\%), o que aponta para um interesse crescente na problematização do padrão de articulação desses elementos ao interior do SUS, sendo destacadas temáticas novas como a dominância financeira na assistência à saúde.

No que se refere à análise da abordagem metodológica (Tabela 3), chama a atenção que a maioria dos trabalhos foi classificada na categoria "outros" $(24,7 \%)$, face à multiplicidade de escolhas metodológicas. Tais estudos apenas descrevem o uso de técnicas como entrevistas e análise documental, sem, contudo, especificar a estratégia da pesquisa. Há ainda aqueles que não explicitam nem a metodologia nem as técnicas utilizadas $(26,4 \%)$. Destacam-se, também, os 9,0\% de estudos de revisão e o fato de $25,4 \%$ terem sido classificados na categoria "não se apli-

Tabela 2. Número e percentual de textos publicados na Revista Ciência \& Saúde Coletiva sobre políticas de saúde segundo tema de publicação (1996-2019).

\begin{tabular}{|c|c|c|}
\hline Temas & No & $\%$ \\
\hline \multicolumn{3}{|l|}{$\begin{array}{l}\text { Análise de Políticas de Saúde } \\
\text { Específicas }\end{array}$} \\
\hline $\begin{array}{l}\text { Políticas voltadas a grupos } \\
\text { populacionais específicos }\end{array}$ & 30 & 7,6 \\
\hline $\begin{array}{l}\text { Políticas voltadas para o } \\
\text { enfrentamento de problemas } \\
\text { específicos }\end{array}$ & 95 & 23,9 \\
\hline Subtotal & 125 & 31,5 \\
\hline \multicolumn{3}{|l|}{ Análise Política em Saúde } \\
\hline Reforma Sanitária Brasileira & 24 & 6,0 \\
\hline $\begin{array}{l}\text { Política de saúde em uma } \\
\text { perspectiva internacional }\end{array}$ & 21 & 5,3 \\
\hline Processo de construção do SUS & 43 & 10,9 \\
\hline Relações entre o público e privado & 24 & 6,0 \\
\hline Subtotal & 112 & 28,2 \\
\hline \multicolumn{3}{|l|}{ Componentes do Sistema de Saúde } \\
\hline Ciência, tecnologia e inovação & 16 & 4,0 \\
\hline Financiamento da saúde & 23 & 5,8 \\
\hline Gestão de sistemas & 52 & 13,1 \\
\hline Modelos de atenção em saúde & 34 & 8,6 \\
\hline Participação e controle social & 14 & 3,5 \\
\hline Recursos humanos em saúde & 21 & 5,3 \\
\hline Subtotal & 160 & 40,3 \\
\hline Total & 397 & 100,0 \\
\hline
\end{tabular}

Fonte: Revista Ciencia \& Saúde Coletiva. Homepage Scielo. Disponível em https://www.scielo.br/scielo.php?script=sci_ serial\&pid $=1413-8123 \& \operatorname{lng}=e n$ 
Tabela 3. Número e percentual de textos publicados na Revista Ciência \& Saúde Coletiva sobre políticas de saúde segundo abordagem metodológica (1996-2019).

\begin{tabular}{lrc}
\multicolumn{1}{c}{ Metodologia } & No & $\%$ \\
Análise de implantação & 1 & 0,2 \\
Análise da implementação & 7 & 1,8 \\
Análise exploratória & 5 & 1,3 \\
Análise histórica & 3 & 0,8 \\
Ensaio & 6 & 1,5 \\
Estudo comparativo & 2 & 0,5 \\
Estudo de avaliabilidade & 2 & 0,5 \\
Estudo qualitativo & 15 & 3,8 \\
Estudo de caso & 11 & 2,8 \\
Estudo de casos múltiplos & 5 & 1,3 \\
Revisão & 36 & 9,0 \\
Outros & 98 & 24,7 \\
Metodologia não especificada & 105 & 26,4 \\
Não se aplica & 101 & 25,4 \\
Total & 397 & 100,0
\end{tabular}

Fonte: Revista Ciencia \& Saúde Coletiva. Homepage Scielo. Disponível em https://www.scielo.br/scielo.php?script=sci_ serial\&pid=1413-8123\&lng=en

ca" por não requererem a adoção de uma dada metodologia científica.

Quanto à análise dos referencias teóricos, a maioria das publicações (61\%) não explicita aqueles que teriam sido utilizados, o que confirma a crítica comentada na literatura acerca da "rarefação teórica" na área ${ }^{36}$. Entre aqueles que citam um dado referencial teórico, observa-se uma profusão de perspectivas, não sendo possível identificar um enfoque predominante.

Conforme estudo prévio ${ }^{37}$, a análise dos referenciais identificou um conjunto heterogêneo de enfoques teóricos e autores mobilizados pelos artigos. Entre os referenciais teóricos citados nos trabalhos revisados $(9 \%)$, podemos identificar uma filiação a teorias de matrizes sociológicas contemporâneas (Bourdieu, Habermas, Foucault); teorias do Planejamento na América Latina; teorias da ciência política; teorias de médio alcance; modelos teóricos de análise política; teorias das organizações/gestão; e autores da Saúde Coletiva.

Podemos considerar, todavia, que esse conjunto de abordagens teóricas mobilizadas nos estudos reconfiguram em diferentes períodos quatro tradições sociológicas: uma tradição crítica ou do conflito, uma tradição racional-utilitarista, uma tradição funcionalista e outra microintera- cionista $^{38}$. Esta profusão de enfoques, mobilizados para analisar diferentes objetos mostra certo afastamento de uma perspectiva macro, de ancoragem marxista, utilizada para abordar as tensões da relação Estado e sociedade, outrora encontrada na produção de pesquisas do início da década de $1990^{33,39}$.

As diferentes classificações temáticas e metodológicas, feitas em artigos da Revista dificultam a comparação. Frequentemente os artigos sobre políticas são analisados conjuntamente no interior da rubrica Política, planejamento e gestão ${ }^{14,40}$.

\section{A Política de Saúde e a Saúde Coletiva}

A delimitação do espaço da análise das políticas de saúde, em um periódico, não é simples, tendo em vista as diversas definições sobre o termo e suas múltiplas intersecções com outras disciplinas e áreas temáticas. Assim, as políticas de saúde são referidas não apenas às análises sobre as ações estatais direcionadas aos problemas de saúde, mas a diversos estudos sobre avaliação de serviços de saúde, sistemas de saúde, financiamento, relações público-privadas, entre outros. Assim, segundo classificação obtida na biblioteca SciELO/Saúde Pública, a temática sobre políticas de saúde estava classificada em rubrica diferente daquela do "SUS" e de "Planejamento e gestão"

Se as primeiras publicações da Abrasco estavam voltadas para sumarizar seus encontros e introduzir as discussões principalmente sobre a formação de recursos humanos e secundariamente sobre a pesquisa, a C\&SC inicia trazendo para o centro o debate sobre as políticas de saúde.

Antes da criação da Abrasco, porém, foi fundada, no ano de 1975, a International Association of Health Policy (IAHP), da qual participaram diversos brasileiros. Essa associação estabeleceu diálogos com a Abrasco e a Associação Latinoamericana de Medicina Social (Alames) ${ }^{42}$.

No entanto, Política de Saúde, enquanto disciplina acadêmica desenvolveu-se no Brasil de um modo distinto dos Estados Unidos e da Europa. O fato de sua produção seminal iniciar-se na década de setenta, ao lado de um movimento político de democratização da saúde e da busca de referenciais da Medicina Social do século XIX, pode ajudar a entender parte das suas dificuldades científicas.

Antes da década de setenta, a Saúde Pública no Brasil não contemplava a Política de Saúde, enquanto disciplina. Reconhecia a Administração Hospitalar e a Administração Sanitária, incorporando depois o Planejamento de Saúde. Possivel- 
mente, o marco dessa disciplina no Brasil tenha como referência uma tese defendida em $1972^{43}$. Nos estudos seminais de Cecília Donnangelo ${ }^{43,44}$, embora seja referida a contribuição de Marshall ${ }^{45}$ acerca do reconhecimento dos direitos civis, políticos e sociais na Europa nos séculos XVIII, XIX e $\mathrm{XX}$, respectivamente, a abordagem desenvolvida pela autora remete à dinâmica do capitalismo e à luta de classes para a explicação da intervenção do Estado no que tange ao direito à saúde.

Nesse mesmo ano de 1972 ocorreu a Reunião de Cuenca sobre ciências sociais e saúde e foi publicado um estudo sobre educação médica na América Latina, por um médico e sociólogo ${ }^{46}$, em que o autor explicitava um marco teórico para a investigação. Ainda naquela década foram produzidos mais alguns textos "clássicos" por economistas, sociólogos, psicólogos e médicos ${ }^{47-50}$, contemplando a previdência, políticas de saúde e instituições médicas, assim como um guia para a formulação de políticas de saúde ${ }^{51}$. Do mesmo modo, alguns livros muito divulgados na época traziam para o debate temas de políticas de saú$\mathrm{de}^{52-54}$.

Participantes dessas iniciativas fundaram o Centro Brasileiro de Estudos de Saúde (Cebes) e a Abrasco na mesma década. No âmbito estatal, o governo Geisel estabeleceu como tema da V CNS o Sistema Nacional de Saúde ${ }^{55}$ e da VI CNS a Política Nacional de Saúde ${ }^{56}$. Todavia, muitos dos trabalhos produzidos no período pareciam ser vistos como contribuições para discussão, não como produtos científicos.

Essa área de estudos e de produção de conhecimentos denominada de Política de Saúde foi reconhecida como disciplina acadêmica e âmbito de intervenção social, tendo sido ressaltadas as dimensões de politics e de policy, bem como algumas concepções subjacentes às definições de políticas de saúde ${ }^{57}$. Adotou-se a definição de Política de Saúde como ação ou omissão do Estado, enquanto resposta social, diante dos problemas de saúde e seus determinantes, bem como da produção, distribuição e regulação de bens e serviços ${ }^{39,58,59}$. Admitia-se, enfim, que Política de Saúde deveria ser entendida como integrante de um campo científico (a Saúde Coletiva), como técnica de análise e de formulação de política (policy) e como práxis (politics) ou ação política dos atores sociais ${ }^{57}$.

As observações acima podem auxiliar a compreensão de certas ambiguidades dessa área disciplinar ao envolver objetos de análise, pesquisa e intervenção, de um lado, e o esforço de delimitação conceitual e de construção teórico-metodo- lógico, de outro. Os resultados obtidos pelo presente estudo podem reforçar o reconhecimento de tais ambiguidades, tendo em conta as limitações das diversas classificações utilizadas.

\section{A Revista e a construção do campo}

A construção de um campo é um processo complexo que ocorre em diversos âmbitos do espaço social ${ }^{31}$, sendo que os periódicos científicos fazem parte desse processo. No caso específico da SC, diversas revistas contribuíram a esse respeito veiculando a produção acadêmica, debates sobre as políticas e outros produtos ${ }^{16}$. Nos anos 1990, existiam 12 revistas nacionais onde a produção científica da SC poderia ser veiculada: A Revista de Saúde Pública da USP, criada em 1967; a Revista Brasileira de Saúde Ocupacional publicada, desde 1973, pela Fundacentro; a Revista Baiana de Saúde Pública da Secretaria de Saúde do Estado da Bahia (SESAB) de 1974; a Revista Saúde em Debate, ligada ao Cebes e criada em 1976; Os Cadernos de Saúde Pública (CSP) da ENSP, em 1985; os Cadernos de Saúde Coletiva (CSC) da UFRJ, em 1987; a Physis vinculada à UERJ, em 1991; a Revista Saúde e Sociedade (RSS) da FSP/ USP e o Informe Epidemiológico do SUS (IESUS), ambos em 1992; este último, transformado em 2003 na Revista Epidemiologia e Serviços de Saúde (RESS), editada pelo Ministério da Saúde; a Revista História, Ciências, Saúde- Manguinhos (HCSM), publicada pela Casa de Oswaldo Cruz, da Fundação Oswaldo Cruz - Fiocruz desde 1994; a C\&SC em 1996; e a Interface de 1997.

Se a RSP reivindicava, quando da sua criação, sua continuidade em relação à Higiene que a antecedeu ${ }^{60}$, outras traduzem, em alguma medida, nos editoriais inaugurais, sua relação com a construção da SC. Os CSP se apresentam como revista voltada para o campo da Saúde Pública ${ }^{61}$. Por outro lado, a Interface nasce enfatizando "a busca de articulação entre os paradigmas das ciências biológicas e sociais" ${ }^{62}$. Também a Revista HCS Manguinhos destaca que o periódico reflete o propósito da Fiocruz de compreender a saúde como o ponto de encontro entre as “... ciências duras e ciências sociais, do laboratório e da política, da ciência e da sociedade"63. Já as revistas Physis e a C\&SC tematizam explicitamente a novidade da SC, não somente incorporando a expressão nos seus títulos, mas analisando teoricamente as diferenças com a Saúde Pública ${ }^{19,64}$.

O espaço das revistas nucleares para a construção da SC assim estava delineado, abrigando os diferentes grupos que fizeram parte desse pro- 
cesso: epidemiologistas, planejadores, cientistas sociais e instituições centrais na constituição do campo: USP, IMS/UERJ, UFRJ, ENSP e UFBa. A Revista Saúde em Debate se propunha a ser um espaço para a discussão sobre as relações entre a saúde e a estrutura social, mas que também reivindicava, no primeiro número, a identidade com algumas revistas da Higiene e da Saúde Pública que lhe antecederam ${ }^{65}$. Havia ainda a Revista Baiana de Saúde Pública e o IESUS que embora ligados a órgãos governamentais, divulgaram artigos científicos e debates especializados. Enquanto as demais revistas faziam parte do campo científico ou com conexões com instituições administrativas, a revista do Cebes ocupava claramente a posição política de crítica ao sistema de saúde e veiculadora de propostas para a Reforma Sanitária Brasileira.

Voltada para a construção do campo da SC, a C\&SC diferenciava-se das demais por ter sido criada ao interior da Abrasco e de ter como objetivos explícitos a contribuição para a "...divulgação e reflexão crítica da história e da memória do campo..." 19 .

A análise dos artigos revisados permite concluir que a Revista abriu espaço para a reflexividade sobre a SC. Assim, a indagação sobre o significado da SC, suas relações com a Saúde Pública, suas especificidades e superposições com outras disciplinas foram tematizadas ${ }^{13,14,66}$.

Cabe destaque a reflexão teórica/epistemológica do campo veiculada por análises e debates sobre transdisciplinaridade ${ }^{67-69}$. Essa discussão é central na constituição da SC tendo em vista que as tensões relacionadas com a delimitação da sua especificidade e afirmação como um novo espaço social fizeram parte da sua história. Outros temas centrais à constituição do campo foram retomados, a exemplo da discussão sobre o direito à saúde ${ }^{70,71}$, além da discussão sobre o próprio conceito de coletivo ${ }^{72}$.

O ponto de vista sobre o significado da SC, segundo o qual este campo pode ser considerado como sinônimo da Saúde Pública, possivelmente relacionado com a sua incorporação em importantes instituições e também vinculado ao movimento de busca de sua internacionalização ${ }^{73}$, pode ser identificado em alguns marcos ao longo dessa trajetória. Se no seu primeiro número, a C\&SC coloca como seu objetivo contribuir para a constituição do campo da SC, quatro anos depois, no número comemorativo dos 100 anos da Saúde Pública, o editorial situa o SUS como parte do desenvolvimento da Saúde Pública institucionalizada ${ }^{14}$. Da mesma forma, na comemoração dos 20 anos predominam as referências à Saúde Pública $^{14}$. A participação nas disputas do campo científico também introduz como questão central da Revista a sua internacionalização ${ }^{74}$.

Ainda nessa linha, análises e avaliações sobre o desenvolvimento da Pós-Graduação foram mostrando a consolidação da SC como universo relativamente autônomo, tanto no que diz respeito à formação de recursos humanos especializados como no que concerne à produção de conhecimento específico ${ }^{17,75,76}$ a despeito do reconhecimento da sua diversidade ${ }^{77}$. A evolução dos programas de Pós-Graduação foi analisada em diversos momentos ${ }^{17,71,78,79}$, inclusive os efeitos dessa consolidação no SUS, na capacitação de profissionais nas áreas de gestão e epidemiologia, como na translação do conhecimento para a prática dos serviços de saúde ${ }^{80}$. A análise da demanda aos programas de Pós-Graduação em 1996 mostrou a importância dos mestrados para a qualificação de profissionais oriundos dos serviços de saúde e do doutorado para formação de docentes e pesquisadores ${ }^{81}$. A inserção desses profissionais, dez anos depois é majoritariamente no setor público, nos três níveis de governo ${ }^{82}$.

Da mesma forma a criação dos cursos de graduação em SC correspondeu a uma afirmação da especificidade da área no que diz respeito ao exercício de um conjunto de práticas voltadas para a saúde no âmbito populacional ${ }^{83}$.

A Revista também contribuiu para a veiculação das posições que têm polarizado a SC nesses 25 anos a exemplo do debate sobre o que seria a verdade no campo científico da saúde onde, por um lado situa-se a medicina e a saúde baseada em evidências, e por outro os estudos qualitativos ${ }^{84}$.

Por outro lado, a despeito das reiteradas afirmações da ociosidade do debate qualitativo $\mathrm{x}$ quantitativo, a Revista deu ênfase à importância dos estudos qualitativos, como novo paradigma em contraposição ao denominado modelo biomédico ${ }^{85}$.

A polêmica envolvendo as normas de ética na pesquisa qualitativa também fez aparecer a oposição entre o biomédico e as ciências humanas e sociais. Em alguns momentos nessa discussão a SC não aparece como campo transdisciplinar entre a medicina e as ciências sociais, mas como multidisciplinar sendo que os pesquisadores das ciências humanas e sociais recorrem aos fóruns dessa área, e não da SC, para defender a especificidade desse subcampo ${ }^{14,86}$.

Também abrigou discussões e análises sobre os três subcampos constitutivos da SC: a Epidemiologia e seu desenvolvimento na América La- 
tina $^{87,88}$; as Ciências Sociais em Saúde ${ }^{16,36}$; e a área do Planejamento, gestão e avaliação em saúde ${ }^{89}$.

Essa terceira área temática que também pode ser analisada como um subcampo da $\mathrm{SC}^{15}$, teve diversas denominações ao longo dos últimos 40 anos, variações estas que correspondem ao processo de construção do campo e também do SUS. A despeito de ser inicialmente denominada de "Administração e Planejamento em Saúde"90 ou "Planejamento \& Gestão em Saúde" e posteriormente de "Planejamento, gestão, avaliação em saúde"36, sempre incorporou entre seus objetos o estudo das políticas de saúde. Foi também considerada eminentemente como um "campo de práticas" 92 .

Ainda abriu espaço para temas com maior ou menor grau de delimitação relacionados à construção do SUS que viriam a se constituir em grupos temáticos da Abrasco. Alguns desses grupos evoluíram na construção da sua identidade e buscando aumentar sua autonomia relativa, enquanto subespaço social ao interior da SC ou mesmo na interface com outras disciplinas ou profissões, a exemplo da Saúde Bucal Coleti$\mathrm{va}^{93,94}$, Saúde e ambiente ${ }^{95}$, Informação em saú$\mathrm{de}^{96}$, Avaliação de Programas de Saúde ${ }^{97}$. Alguns se autodenominaram de campos, a exemplo da Assistência Farmacêutica ${ }^{98}$.

A Revista constituiu-se, ainda, em espaço para a discussão dos diversos pontos de vista sobre as políticas de saúde. O formato debate, com um texto principal e a seleção de diversos comentadores com pontos de vista por vezes diferentes, pode ser considerado como parte do processo de construção social do campo. A guisa de exemplo, pode-se ilustrar com o primeiro número onde os diversos pontos de vista sobre a Reforma Sanitária e as Políticas de Saúde na conjuntura podem ser recuperados daquele debate inaugural. Unificados em torno da crítica às políticas neoliberais e da defesa da universalidade e integralidade, três pontos de vista sobre a Reforma Sanitária podem ser apreendidos daqueles artigos: o SUS como parte da reforma administrativa do Estado ${ }^{99,100}$, o SUS democrático da Reforma Sanitária e o movimento sanitário ${ }^{101-103}$ e um dos pontos de vista das ciências sociais: tanto como análise de políticas de saúde ${ }^{104}$ como análise política das políticas e também sociológica ${ }^{69}$.

A reflexão sobre as políticas de saúde acompanhou as diferentes conjunturas, a exemplo da discussão nos anos $1990^{105}$ que mapeou "avanços" e "dificuldades". Destacam-se ainda os eventos comemorativos em que se apresentam balanços sobre o desempenho do SUS, como aquele relativo aos 20 anos do SUS ${ }^{106}$ e o relacionado aos 30 anos do SUS ${ }^{107}$.

\section{Comentários finais}

A C\&SC constituiu-se em espaço para a construção da SC em múltiplos âmbitos, particularmente no que diz respeito à reflexividade sobre campo, por meio da análise semântica, histórica e sociológica dos distintos sentidos, bem como das suas principais características. Também abrigou a reflexão teórico-epistemológica-metodológica sobre a constituição de seus subcampos principais e áreas temáticas.

Especificamente em relação às políticas de saúde, a despeito do pequeno percentual de artigos específicos sobre a temática, ela abarcou os diversos significados para o campo: Política de Saúde como disciplina acadêmica e o debate sobre as políticas de saúde. Essa própria delimitação traduz as disputas do campo e o processo de construção social. Varia possivelmente a depender da posição ocupada pelo autor e sua trajetória ao interior da SC.

Todavia, as limitações do presente estudo são de várias ordens: no que diz respeito à análise das políticas, a inexistência de consenso sobre as classificações temáticas dificulta a comparação com outros trabalhos; também a análise do conteúdo completo dos artigos poderia trazer outros elementos para a interpretação. Já no que diz respeito à investigação sobre as contribuições da Revista para com a construção do campo faz falta o exame da evolução do espaço das revistas que ao lado da C\&SC participaram desse processo. A apreensão das relações entre a C\&SC e a constituição do campo da SC também requereria buscar recuperar o espaço dos pontos de vista sobre as principais questões em disputa ao longo desse percurso, os agentes e as Instituições envolvidos na sua produção, buscando relacionar as posições e as trajetórias às tomadas de posição identificadas e como estas se refletiram nos artigos.

A despeito dessas lacunas, a apreensão aqui feita possibilitou discutir algumas das características e o escopo da produção sobre Política de Saúde nas suas relações com a SC. As transformações evidenciadas na evolução dos objetos e enfoques sobre a temática, desde as suas origens como objeto sociológico até seus desdobramentos com o aporte de outras disciplinas ao lado da diversidade teórico-metodológica, podem estar relacionadas com o processo histórico de incorporação desse objeto nos diversos âmbitos de constituição do campo da SC. 


\section{Colaboradores}

LM Vieira-da-Silva e MA Esperidião contribuíram com a concepção e delineamento do artigo, coleta, análise e redação. AS Silveira contribuiu com a coleta, consolidação dos dados e análise. JS Paim contribuiu com a concepção e delineamento do artigo, analise e redação. Todos os autores revisaram e aprovaram a versão final.

\section{Referências}

1. Walt G, ShiffmanJ, Schneider H, Murray SF, Brugha R, Gilson L. 'Doing' health policy analysis: methodological and conceptual reflections and challenges. Health Policy Plan 2008; 23(5):308-317.

2. Bernier NF, Clavier C. Public health policy research: making the case for a political science approach. $\mathrm{He}$ alth Promot Int 2011; 26(1):109 116.

3. Smith JA. The Idea Brokers: Think Tanks and the Rise of the New Policy Elite. New York: The Free Press; 1991.

4. Donnangelo MCF. A Pesquisa em Saúde Coletiva no Brasil - a década de 70. In: Abrasco, editor. Ensino da Saúde Pública, Medicina Preventiva e Social no Brasil. Rio de Janeiro: UERJ, OPS, Fiocruz; 1983. p. 19-35.

5. Costa NR. Ciencias Sociales e Salud, consideraciones sobre el nascimiento del campo de la Salud Colectiva em Brasil. Cuadernos Medico Sociales 1992; (62):3647.

6. Paim JS, Almeida Filho N. Saúde coletiva: uma "nova saúde pública" ou campo aberto a novos paradigmas? Rev Saude Publica 1998; 32(4):299-316.

7. Ribeiro PT. A instituição do campo cientifico da saúde coletiva no Brasil (1975:1978) [dissertação]. Rio de Janeiro: Fiocruz; 1991.

8. Nunes ED. Saúde Coletiva: História de uma idéia e de um conceito. Saúde Soc 1994; 3(2):5-21.

9. Campos GWS. Saúde pública e saúde coletiva: campo e núcleo de saberes e práticas. Cien Saude Colet 2000; 5(2):219-230.

10. Luz MT. Complexidade do Campo da Saúde Coletiva: multidisciplinaridade, interdisciplinaridade, e transdisciplinaridade de saberes e práticas - análise sócio -histórica de uma trajetória paradigmática. Saúde Soc 2009; 18(2):304-311.

11. Bosi MLM. Pesquisa qualitativa em saúde coletiva: panorama e desafios. Cien Saude Colet 2012; 17(3):575586.

12. Leal MB, Camargo Junior KR. Saúde Coletiva em debate: reflexões acerca de um campo em construção. Interface (Botucatu) 2012; 16(40):53-66.

13. Ianni AMZ, Cristiane S, Barboza R, Alves OSF, Viana SDL, Rocha AT, Rocha AT. Os Congressos Brasileiros de Ciências Sociais e Humanas em Saúde da Abrasco: um campo científico em disputa. Cien Saude Colet 2015; 20(2):503-513.

14. Minayo MCS. Disputas científicas que transbordam para o campo da Ética em pesquisa: entrevista com Maria Cecília de Souza Minayo. Cien Saude Colet 2015; 20(9):2693-2696

15. Vieira da Silva LM. O Campo da Saúde coletiva. Gênese, transformações e articulações com a reforma sanitária brasileira. Salvador, Rio de Janeiro: Edufba, Fiocruz; 2018.

16. Nunes ED. A Revista Ciência \& Saúde Coletiva e o processo de institucionalização de um campo de conhecimentos e práticas. Cien Saude Colet 2015; 20(7):1975-1982.

17. Minayo MCS. Pós-graduação em Saúde Coletiva de 1997 a 2007: desafios, avanços e tendências. Cien Saude Colet 2010; 15(4):1897-1907.

18. Viacava F. Produção científica dos cursos de pós-graduação em Saúde Coletiva no período 1998-2006. Cien Saude Colet 2010; 15(4):1977-1988. 
19. Packer AL. Indicadores de centralidade nacional da pesquisa comunicada pelos periódicos de Saúde Coletiva editados no Brasil. Cien Saude Colet 2015; 20(7):1983-1995.

20. Paim JS. Reforma Sanitária Brasileira: contribuição para a compreensão e crítica. Salvador, Rio de Janeiro: EDUFBA, Fiocruz; 2008.

21. Baptista TW, Azevedo CS, Machado CV, organizadores. Políticas, Planejamento e Gestão em Saúde: abordagens e métodos de pesquisa. Rio de Janeiro: Editora Fiocruz; 2015.

22. Asnake M. A importância da publicação científica para o desenvolvimento da saúde pública. Cien Saude Colet 2015; 20(7):1972-1973.

23. Carvalho MS, Coeli CM, Travassos C. Uma breve história de Cad Saude Publica. Cien Saude Colet 2015; 20(7):2007-2012.

24. Antunes JLF, França Júnior I, Andrade MTD, Barata RCB, Monteiro CA. Desafios editoriais da Rev Saude Publica. Cien Saude Colet 2015; 20(7):1997-2006.

25. Camargo Júnior, KR. Celebrando Ciência e Saúde Coletiva, lembrando da trajetória da Physis. Cien Saude Colet 2015; 20(7):2053-2058.

26. Cyrino AP, Lima EA, Garcia VL, Teixeira RR, Foresti MCPP, Schraiber LB. Um espaço interdisciplinar de comunicação científica na Saúde Coletiva: a Revista Interface (Botucatu). Cien Saude Colet 2015; 20(7):2059-2068.

27. Amarante P, Rizzotto MLF, Costa AM. Memória de um movimento: a Revista Saúde em Debate e a reforma sanitária brasileira. Cien Saude Colet 2015; 20(7):2023-2029.

28. Garcia LP, Duarte E. Epidemiologia e Serviços de Saúde: a trajetória da revista do Sistema Único de Saúde do Brasil. Cien Saude Colet 2015; 20(7):2081-2090.

29. Martins CL, Ribeiro H, Alvarenga AT, Carvalheiro JR. Saúde e Sociedade: parceria e abertura para novas abordagens. Cien Saude Colet 2015; 20(7):2069-2080

30. Jackson Filho JM, Algranti E, Saito CA, Garcia EG. Da segurança e medicina do trabalho à Saúde do Trabalhador: história e desafios da Revista Brasileira de Saúde Ocupacional. Cien Saude Colet 2015; 20(7):2041-2051.

31. Bourdieu P. As regras da arte. Gênese e estrutura do campo literário. Lisboa: Editorial Presença; 1992.

32. Bourdieu P. Sociologie Generale. In: Bourdieu P. Cours au Collège de France, 1981-1983. Paris: Raisons d'agir; 2015. (Vol. 1). p. 440-634.

33. Santos JS, Teixeira CF. Política de saúde no Brasil: produção científica 1988-2014. Saúde debate 2016; 40(108):219-230.

34. Paim JS. Pósfacio - Análise política em saúde: um pensamento estratégico para a ação estratégica. In: Federico L. Análise política em saúde: a contribuição do pensamento estratégico. Salvador: EDUFBA; 2015. p. 279-286.

35. Teixeira CT, Silveira P, organizadores. Glossário de análise política em Saúde. Salvador: Edufba; 2016.

36. Schraiber LB, Peduzzi M, Sala A, Nemes MIB, Castanhera ERL, Kon R. Planejamento, gestão e avaliação em saúde: identificando problemas. Cien Saude Colet 1999; 4(2):221-242.

37. Esperidião MA. Análise política em saúde: síntese das abordagens teórico-metodológicas. Saúde debate 2018; 42(n. esp. 2):341-360.
38. Collins R. Quatro Tradições Sociológicas. Petrópolis: Vozes; 2009.

39. Paim JS, Teixeira CF. Política, planejamento e gestão em saúde: balanço do estado da arte. Rev Saude Publica 2006; 40(n. esp.):73-78.

40. Loyola MAR. A Saga das Ciências Sociais na área da Saúde Coletiva: elementos para reflexão. Physis 2008; 18(2):251-275

41. Minayo MCS, Gomes R. Significância e métrica do que é produzido pela Ciência \& Saúde Coletiva. Cien Saude Colet 2017; 22(2):336-336.

42. International Association of Health Policy (IAHP). Equity on Health Across The World: Neoliberalism or New Welfare Policies? Perugia: X Conference; 1998.

43. Donnangelo MCF. Medicina e sociedade: o médico e seu mercado de trabalho. São Paulo: Pioneira; 1975.

44. Donnangelo MCF, Pereira L. Saúde e Sociedade. São Paulo: Duas Cidades; 1976.

45. Marshall TH. Cidadania, Classe Social e Status. Zahar Ed.: Rio de Janeiro: Zahar; 1967.

46. Garcia JC. La educación medica em la América Latina. Washington: OPS; 1972. (Publicación científica, 255)

47. Braga JCDS. A Questão da Saúde no Brasil - Um estudo das políticas sociais em saúde pública e medicina providenciaria no desenvolvimento capitalista. [dissertação]. Campinas: Unicamp; 1978.

48. Luz MT. As instituições médicas no Brasil - instituição e estratégia de hegemonia. Rio de Janeiro: Graal; 1979.

49. Oliveira JA, Teixeira SMF. A (im) previdência social brasileira: 60 anos de história da Previdencia Social no Brasil. Petrópolis, Rio de Janeiro: Vozes, Abrasco; 1986.

50. Braga JCS, Goes de Paula S. Saúde e Previdência: Estudos de Política Social. São Paulo: Hucitec; 1981.

51. Centro Panamericano de Planificación de la Salud. Formulación de Políticas de Salud. Santiago: OPS: 1975.

52. Mello CG. Saúde e Assistência Médica no Brasil. São Paulo: Cebes-Hucitec; 1977.

53. Guimarães R, organizador. Saúde e Medicina no Brasil: contribuição para um debate. Rio de Janeiro: Edições Graal; 1978.

54. Berlinger G. Medicina e Política. São Paulo: Cebes-Hucitec; 1978.

55. Brasil. Ministério da Saúde (MS). Relatório da V Conferência Nacional de Saúde. Brasília: MS; 1975.

56. Brasil. Ministério da Saúde (MS). Relatório da VI Conferência Nacional de Saúde. Brasília: MS; 1977.

57. Paim JS. Políticas de Saúde no Brasil. In: Rouquayrol MZ, Almeida-Filho N, organizadores. Epidemiologia \& Saúde. $6^{\mathrm{a}}$ ed. Rio de Janeiro: MEDSI; 2003. p. 587603.

58. Paim JS. Saúde e Políticas Públicas. In: Paim JS. Saúde, Política e Reforma Sanitária. Salvador: CEPS-ISC; 2002. p. 383-405.

59. Fleury S, Ouverney AM. Política de Saúde: uma política social. In: Giovanella L, Escorel S, Lobato LVC, Noronha JC, Carvalho AI, organizadores. Políticas e Sistema de Saúde no Brasil. Rio de Janeiro: Editora Fiocruz; 2008. p. 23-64.

60. Paula Souza R. Editorial. Rev Saude Publica 1967; $1(1): 1-2$

61. Sousa AFG. Editorial. Cad Saude Publica 1985; 1(1):00. 
62. Apresentação. Interface (Botucatu) 1997; 1(1):5-5

63. Gadelha P. Apresentação. História, Ciências, Saúde -Manguinhos 1994; 1(1):3-3.

64. Birman J. A physis da saúde coletiva. Physis 1991; 1:1.

65. Editores. Saúde em Debate. Revista do Centro Brasileiro de Estudos em Saúde 1976; 1(1):3-3.

66. Nunes ED. Editorial. Cien Saude Colet 2000; 5(2):216217.

67. Almeida Filho N. Transdisciplinaridade e Saúde Coletiva. Cien Saude Colet 1997; 2(1-2):5-20.

68. Castiel LD. Debate sobre o Artigo de Almeida-Filho: Transdisciplinaridade e Saúde Coletiva. Cien Saude Colet 1997; 2(1-2):27-30.

69. Cohn A. Estado e sociedade e as reconfigurações do direito à saúde. Cien Saude Colet 2003; 8(1):9-18.

70. Debatedores. Cien Saude Colet 2003; 8(1):19-32.

71. Minayo MCS. Pós-Graduação em Saúde Coletiva: Um Projeto em Construção. Cien Saude Colet 1997; 2(12):53-71.

72. Guimarães CF, Silva RAN. Notas para a problematização do coletivo no campo da saúde. Cien Saude Colet 2005; 20(3):913-924.

73. Paim JS. A Reforma Sanitária Brasileira e a Saúde Coletiva: concepções, posições e tomadas de posição de intelectuais fundadores. In: Vieira da Silva LM, organizador. O Campo da Saúde Coletiva. Gênese, transformações e articulações com a Reforma Sanitária Brasileira. Salvador, Rio de Janeiro: Edufba, Fiocruz; 2018.p. 192-221.

74. Campos GWS, Onocko-Campos RT, del Barrio LR. Políticas e práticas em saúde mental: as evidências em questão. Cien Saude Colet 2003; 18(10):2797-2805.

75. Barata R, Santos RV. Pós-graduação em Saúde Coletiva no Brasil: o imprescindível papel da avaliação. Cien Saude Colet 2010; 15(4):1908-1909.

76. Luz MT, Mattos R. Dimensões qualitativas na produção científica, tecnológica e na inovação em Saúde Coletiva. Cien Saude Colet 2010; 15(4):1945-1953.

77. Carvalheiro JR. Janus bifronte e a pós-graduação. Cien Saude Colet 2010; 15(4):1910-1913.

78. Nunes ED. Saúde Coletiva: Revisitando a sua História e os Cursos de Pós-Graduação. Cien Saude Colet 1996; 1(1):55-69.

79. Nunes ED, Ferreto LE, Barros NF. A pós-graduação em Saúde Coletiva no Brasil: trajetória. Cien Saude Colet 2010; 15(4):1923-1934.

80. Novaes HMD, Werneck GL, Cesse EAP, Goldbaum M, Minayo, MCS. Pós-Graduação senso estrito em Saúde Coletiva e o Sistema Único de Saúde. Cien Saude Colet 2018; 23(6):2017-2025.

81. Tanaka ACA. Perfil da Demanda aos Cursos de PósGraduação, de 1996, na Área de Saúde Coletiva. Cien Saude Colet 1997; 2(1-2):108-116.

82. Gomes MHA, Goldenberg P. Retrato quase sem retoques dos egressos dos programas de pós-graduação em Saúde Coletiva, 1998-2007. Cien Saude Colet 2010; 15(4):1989-2005.

83. Bosi MLM, Paim JS. Graduação em Saúde Coletiva: limites e possibilidades como estratégia de formação profissional. Cien Saude Colet 2010; 15(4):2029-2038.

84. Minayo MCS. A busca da verdade no campo científico da saúde. Cien Saude Colet 2013; 18(10):2806-2808.
85. Baixinho CL, Presado MH, Ribeiro J. Investigação qualitativa e transformação da saúde coletiva. Cien Saude Colet 2019; 24(5):1582-1582.

86. Guerriero ICZ, Bosi MLM. Ética em pesquisa na dinâmica do campo científico: desafios na construção de diretrizes para ciências humanas e sociais. Cien Saude Colet 2015; 20(9):2615-2624.

87. Barata RB, Barreto ML. Algumas Questões sobre o Desenvolvimento da Epidemiologia na América Latina. Cien Saude Colet 1996; 1(1):70-79.

88. Turci SRB, Guilam MCR, Câmara MCC. Epidemiologia e Saúde Coletiva: tendências da produção epidemiológica brasileira quanto ao volume, indexação e áreas de investigação - 2001 a 2006. Cien Saude Colet 2010; 15(4):1967-1976.

89. Levcovitz E, Lima LD, Machado CV. Política de saúde nos anos 90: relações intergovernamentais e o papel das Normas Operacionais Básicas. Cien Saude Colet 2001; 6(2):269-291.

90. Associação Brasileira de Pós-Graduação em Saúde Coletiva (Abrasco). Ensino da saúde pública, medicina preventiva e social no Brasil. Rio de Janeiro: Abrasco; 1982.

91. Teixeira CF, Sá MC. Planejamento \& Gestão em Saúde: Situação Atual e Perspectivas para a Pesquisa, o Ensino e a Cooperação Técnica na Area. Cien Saude Colet 1996; 1(1):80-103.

92. Luz MT. A Produção Científica em Saúde Coletiva (1994-1995). Cien Saude Colet 1997; 2(1-2):117-141.

93. Soares CLM, Paim JS, Chaves SCL, Rossi TRA, Barros SG, Cruz DN. O movimento da Saúde Bucal Coletiva no Brasil. Cien Saude Colet 2017; 22(6):1805-1816.

94. Celeste RK, Warmling CM. Produção bibliográfica brasileira da Saúde Bucal Coletiva em periódicos da saúde coletiva e da odontologia. Cien Saude Colet 2014; 19(6):1921-1932.

95. Augusto LGS, Tambellini AT, Miranda AC, Carneiro FF, Castro H, Porto MFS, Schütz GE. Desafios para a construção da "Saúde e Ambiente" na perspectiva do seu Grupo Temático da Associação Brasileira de Saúde Coletiva. Cien Saude Colet 2014; 19(10):4081-4089.

96. Moraes IHS, Santos SRFR. Informação em Saúde: Os Desafios Continuam. Cien Saude Colet 1998; 3(1):3751.

97. Hartz ZMA, Pouvourville G. Avaliação dos Programas de Saúde: A Eficiência em Questão. Cien Saude Colet 1998; 3(1):68-82.

98. Osorio-de-Castro CGS, Oliveira MA, Vasconcelos DMM. Assistência Farmacêutica: um campo em consolidação. Cien Saude Colet 2017; 22(8):2432-2432.

99. Barros E. Política de Saúde no Brasil: a Universalização Tardia como Possibilidade de Construção do Novo. Cien Saude Colet 1996; 1(1):5-17.

100. Junqueira LAP. A Descentralização e a Gestão Municipal da Política de Saúde. Cien Saude Colet 1996; 1(1):21-22.

101. Campos GWS. Papel do Movimento Sanitário na Construção do Novo. Cien Saude Colet 1996; 1 (1):2325.

102. Paim JS. Políticas de Saúde no Brasil ou Recusando o Apartheid Sanitário. Cien Saude Colet 1996; 1(1):1820. 
103. Rodrigues NE. Comentários a: "A Política de Saúde no Brasil a Universalização Tardia como Possiblidade de Construção do Novo". Cien Saude Colet 1996; 1(1):26-27.

104. Costa NR. Políticas Sociais e Crise do Estado Relativizando Crenças Cognitivas. Cien Saude Colet 1996; $1(1): 28-34$.

105. Goulart FAA. Esculpindo o SUS a golpes de portaria... - considerações sobre o processo de formulação das NOBs. Cien Saude Colet 2001; 6(2):294-298.

106. Vaitsman J, Moreira MR, Costa NR. Editorial. Cien Saude Colet 2009; 14(3):690.

107. Paim JS, Temporão JG, Penna GO, Santos NR, Pinto LF. Sistema Único de Saúde: 30 anos de luta!. Cien Saude Colet 2018; 23(6):1704.

Artigo apresentado em 23/06/2020

Aprovado em 23/06/2020

Versão final apresentada em 25/06/2020 